\title{
Finding Antibodies that Work
}

\author{
Joseph P Chandler* \\ Maine Biotechnology Services, Inc., Portland, ME
}

Received: November 13, 2013; Accepted: November 14, 2013; Published: November 18, 2013

*Corresponding authors: Joseph P Chandler, Maine Biotechnology Services, Inc., Portland, ME, USA, Tel: 207-797-5454; E-mail: jpchandler@ mainebiotechnology.com

\section{Editorial}

As a new on-line journal, Symbiosis On-line Journal envisions that it will allow its readers to have "access to emerging literature and to promote the knowledge transfer among research communities. " Without doubt, the rate of communication that occurs on-line is exceptionally rapid and can be, at times, voluminous. As immunologists with monoclonal antibodies as our specialty, we do our best to keep abreast of all things antibody. One arena that has compelled our interest of late has been the numerous blogs and forums devoted to "useless" commercial antibodies. The frustration in these forums is palpable. Many in this industry have written compelling defenses and we will be among those who would like to make a few suggestions to alleviate some of this aggravation. We, too, have been on the receiving end of antibodies that did not perform as we had expected. Potential mismatches and frustration can be avoided through adequate preparation and collaboration.

Know your supplier. Thorough knowledge of your target against which the antibody is to react has to be hand-in-hand with how well your supplier knows their products. Large antibody supply houses serve an invaluable service, as they can be a one-stop shop for a variety of antibodies, particularly to the research market. Unfortunately, with thousands of antibodies in their repertoire, it is difficult for any one customer service representative to know each product in detail. On the other hand, the small, often unknown, supplier is more likely the home of the laboratory at which the antibody was developed. That supplier will most often have an extensive packet of information related to their antibody. Also, consider that the antibody that is being marketed by them may not the only one they have. A decision had to be made to sell a small family of products from the many that were generated so in their cryo-storage tanks there are likely several other candidates that can be tested if the ones that are being sold do not work exactly to meet your requirements. All you have to do is ask. Keep in mind that no one supplier will have evaluated any one of its antibodies in all the formats possible. The vendor has a responsibility to be truthful, knowledgeable, and supportive, so if they cannot tell you what immunogenic was used, for example, to generate their antibody - find another source.
Know your target and define a clear goal for the antibody. In essence, define the target, study the choices of available antibodies and validate them. This will determine which product is the best choice, and how much validation is required. Spend the time considering the potential application for the antibody. Selection of the best antibody for different applications is most often mutually exclusive. Is the target the same as it appears in nature? Was the antibody generated against a native molecule, peptide sequence or recombinant protein? Will the target be modified? Formaldehyde fixation or chemical denaturation can cause conformational changes and/or chemical modification to the amino acids within the target molecule. Since monoclonal antibodies recognize a specific epitope in a distinct orientation, these modifications can cause a complete lack of recognition or significantly reduced sensitivity. Correspondingly, an antibody destined to function as capture in a lateral flow assay would ideally have a rapid "on rate" and slow "off rate" to hold the analyze in place for visualization. Antibody-matched pairs should achieve relevant sensitivity and specificity before going to market (an important question to ask the supplier). If the screening strategies implemented during the development phase of the project solely employed the native molecule in solution, the resulting highest-ranking antibodies may not be well suited for some applications if the test sample requires pre-treatment. When selecting an antibody for use in a specific application, it is critical to determine whether the antibody has been validated for use in that particular application to best mitigate the risk when purchasing the reagent.

Having the opportunity to articulate the specific applications, assay formats, sample matrices and treatment conditions will facilitate a more productive collaboration and increase the likelihood of finding products that meet your needs. Unfortunately, antibodies are not interchangeable and must be validated in a particular application prior to use. When at a dead end, though, you still have choices. Most antibody developers are more than willing to develop an antibody that will meet your exact requirement. Our best advice is to work closely with your supplier. Together, we will be able to make the search for the right antibody far less elusive. 